\title{
How Education and Racial Segregation Intersect in Neighborhoods with Persistently Low COVID-19 Vaccination Rates in Philadelphia
}

John Rich ( $\nabla$ jar82@drexel.edu )

Drexel University

Edward Miech

Regenstrief Institute

Usama Bilal

Drexel University

Theodore Corbin

Rush University Medical Center

\section{Research Article}

Keywords: COVID-19 vaccination, residential racial segregation, education, health inequity, configurational comparative methods, coincidence analysis, geospatial analysis, intersectionality.

Posted Date: February 18th, 2022

DOI: https://doi.org/10.21203/rs.3.rs-1332567/v1

License: (a) (i) This work is licensed under a Creative Commons Attribution 4.0 International License. Read Full License 


\section{Abstract}

Background: COVID-19 infection has disproportionately affected socially disadvantaged neighborhoods. Despite this disproportionate burden of infection, these neighborhoods have also lagged in COVID-19 vaccinations. To date, we have little understanding of the ways that various types of social conditions intersect to explain the complex causes of lower COVID-19 vaccination rates in neighborhoods.

Methods: We used configurational comparative methods (CCMs) to study COVID-19 vaccination rates in Philadelphia by neighborhood (proxied by zip code tabulation areas). Specifically, we identified neighborhoods where COVID-19 vaccination rates (per 10,000) were persistently low from March 2021 May 2021. We then assessed how different combinations of social conditions (pathways) uniquely distinguished neighborhoods with persistently low vaccination rates from the other neighborhoods in the city. Social conditions included measures of economic inequities, racial segregation, education, overcrowding, service employment, public transit use, health insurance and limited English proficiency.

Results: Two factors consistently distinguished neighborhoods with persistently low COVID-19 vaccination rates from the others: college education and concentrated racial privilege. Two factor values together - low college education AND low/medium concentrated racial privilege - identified persistently low COVID-19 vaccination rates in neighborhoods, with high consistency (0.92) and high coverage (0.86). Different values for education and concentrated racial privilege - medium/high college education OR high concentrated racial privilege - were each sufficient by themselves to explain neighborhoods where COVID-19 vaccination rates were not persistently low, likewise with high consistency (0.93) and high coverage (0.97).

Conclusions: Pairing CCMs with geospatial mapping can help identify complex relationships between social conditions linked to low COVID-19 vaccination rates. Understanding how neighborhood conditions combine to create inequities in communities could inform the design of interventions tailored to address COVID-19 vaccination disparities.

\section{Background}

The COVID-19 pandemic has revealed many forms of inequity. For example, it is well-documented that Black and Brown communities and poor communities have had higher rates of COVID-19 infection $(1,2)$. Geospatial analyses have uncovered racial and socioeconomic inequities in COVID-19 testing, positivity, confirmed cases and mortality in New York, Philadelphia and Chicago $(3,4)$. These same populations should have been considered as priority populations during the early months of vaccine rollout (5), and this was part of the recommendation of the National Academies of Sciences, Engineering, and Medicine (6). However, the rollout was not spread out evenly, and followed a similar pattern as COVID-19 infections (7-9). For example, COVID-19 vaccination rates in Philadelphia vary widely by neighborhood (10). The situation is similar in other large US cities, with COVID-19 vaccination rates being generally lower in socially disadvantaged neighborhoods (11). 
Correlational analyses looking at spatial inequities have been able to show that factors such as lack of health insurance, poverty and racial segregation are independently associated with lower rates of COVID19 vaccination $(4,12-14)$. However, since social conditions are highly collinear with each other, approaches using the Social Vulnerability Index (SVI), which incorporates multiple social determinants of health into a single measure, have also been applied to local data. $(3,15-17)$. These studies have shown high correlations between the SVI and COVID-19 testing, positivity, confirmed cases and mortality at the neighborhood level (3), and with COVID-19 vaccination rates at the county level (7-9) and the neighborhood level (11). However, the practical application of studies using multidimensional indices is complex because there are multiple variables involved in the creation of these indices (15 in the case of the SVI); and it is not possible to tease out the impact of individual factors.

Ragin and Fiss have proposed addressing the intersectional nature of inequity through using configurational comparative methods (CCMs), which "shift the focus from the separate effects of, for example, race and gender, towards their combined and synergistic effects (18)." CCMs represent a novel approach to understanding complex phenomena. CCMs seek to uncover Boolean conjunctions - the ways in which specific conditions, when they appear together with certain other specific conditions, link directly to important outcomes. CCMs - which include qualitative comparative analysis and coincidence analysis (CNA) - draw on Boolean algebra and set theory to produce configurational models that can complement those generated with more traditional approaches, yielding new and useful insights (19-22).

CCMs are particularly well-suited to understanding geospatial inequities in vaccinations because they are case-based methods which can be applied to a relatively small number of cases, typically between 10 and 100 cases. CCMs can also identify Boolean disjunctions, where multiple pathways lead to the same outcome of interest. These features make CCMs a good fit for analyses focused on exploring and understanding the complex causes of COVID-19 inequities $(19,23-25)$.

In this study, we sought to understand how different combinations of social conditions could help explain COVID-19 vaccination levels at the neighborhood level. Our specific goal was to use CCMs to examine configurations of neighborhood social conditions consistently linked to low COVID-19 vaccination rates in Philadelphia. We used CNA, a new cross-case method to identify the crucial difference-making conditions for the outcome of low COVID-19 vaccination rates $(20,22)$. CCMs, including CNA, are increasingly being used in health services research and implementation and dissemination research (2630).

\section{Methods}

We used data on COVID-19 vaccination rates per 10,000 residents (at least 1 dose) at the zip code tabulation area (ZCTA) level (henceforth, neighborhoods) publicly available from the Philadelphia Department of Public Health on three dates: March 18th, April 18th and May 18th, 2021. For reference, Philadelphia opened eligibility to all adults on April 16th, 2021. We used neighborhood-level data publicly available from the 2014-2018 American Community Survey for sociodemographic variables - \% with 
college education, \% uninsured, \% households with limited English proficiency, \% working in service jobs, $\%$ using public transportation, and \% overcrowded households [ $>1$ person per room]. Based on Krieger (31), we also computed the Index of Concentration at the Extremes for Black non-Hispanic populations and for income, also at the neighborhood level.

\section{Calibration}

We performed multi-value calibration on all factors and the outcome COVID-19 vaccination rates. Vaccination rates were calibrated based on Philadelphia Department of Public Health (PDPH) reporting categories for percent of residents with at least one COVID-19 vaccination. For the three dates March 18, April 18 and May 18, we calibrated neighborhood level COVID-19 vaccination rates based on the tertile categories used by the PDPH to publicly report neighborhood rates per 10,000 residents. For March: $<1500=$ Low, 1501-2000 = Medium, $>2000=$ High; for April: $<2500=$ Low, 2501-3400 = Medium, $>3400$ $=$ High; and for May: $<3400=$ Low, 3401-4500 = Medium, $>4500=$ High. Calibration of social conditions was based on tertiles where values greater than or equal to 67 th percentile were categorized as high, values greater than $33 \mathrm{rd}$ percentile and less than 67 th percentile were categorized as medium, and values less than or equal to 33rd percentile were categorized as low (\% with college education, \% uninsured, \% households with limited English proficiency, racial segregation and economic inequity, \% using public transportation, \% overcrowded households). Because our analysis focused on those neighborhoods with persistently low COVID-19 vaccination rates over the three-month period, our main analysis only includes neighborhoods that had a low value in all three months, resulting in a total of $n=13$ neighborhoods out of a total of 43 in the analysis.

Income Inequities: Index of Concentrations at the Extremes-Income:

To proxy income inequities, we used the Index of Concentration at the Extremes-Income (ICE-Income) measure where negative one $(-1)$ represents the least concentrated economic privilege (extreme concentration of low-income residents) and positive one (1) represents the most concentrated economic privilege (extreme concentration of high-income residents.) We calibrated ICE-Income based on tertiles where values from -1 to -0.23 were coded as zero (low concentrated economic privilege), values from $>-0.23$ to $<0.07$ were coded as one (medium concentrated economic privilege), and values from 0.07 to 1 were coded as two (high concentrated economic privilege).

Racial Segregation: Index of Concentrations at the Extremes-Non-Hispanic Black:

To proxy racial segregation, we used the Index of Concentration at the Extremes-Non-Hispanic Black (ICEBlackNH). ICE-BlackNH values range from negative one $(-1)$ representing the least concentrated racial privilege (extreme concentration of Black non-Hispanic residents) to positive one (1) representing the most concentrated racial privilege (extreme concentration of White non-Hispanic residents). We calibrated ICE-BlackNH based on tertiles where values from -1 to -0.37 were coded as zero (low concentrated racial privilege), values from $>-0.37$ to $<0.47$ were coded as one (medium concentrated racial privilege), and values from 0.47 to 1 were coded as two (high concentrated racial privilege). 
Health Insurance:

Percentage of residents without health insurance (range 2.7-13.8\%) was calibrated based on tertiles where values from $2.7-6.5 \%$ were coded as zero (low uninsured), values $>6.5 \%-9.7 \%$ were coded as one (medium uninsured), and values $>9.7 \%$ were coded as two (high uninsured).

\section{Education:}

Percentage of residents with college education ranged from 4.5-85.6\%. We calibrated this condition based on tertiles where values $\leq 20 \%$ were coded as zero (low college education), values $>20 \%$ and $40.1 \%$ were coded as one (medium college education), and values $>40.1 \%$ were coded as two (high college education).

\section{Limited English Proficiency:}

Percentage of households with limited English proficiency ranged from $0.4-25.1 \%$. We calibrated limited English proficiency based on tertiles where values $\leq 2.2 \%$ were coded as zero (low limited English proficiency), values between $2.2 \%$ and $4.7 \%$ were coded as one (medium limited English proficiency), and values $>4.7 \%$ were coded as two (high limited English proficiency).

Public Transportation Use:

Percentages of residents using public transportation by neighborhood ranged from $9.2-45.3 \%$. We calibrated public transportation use based on tertiles where values $\leq 21.4 \%$ were coded as zero (low public transportation use), values between $21.4 \%$ and $29.5 \%$ were coded as one (medium public transportation use), and values $>29.5 \%$ were coded as two (high public transportation use).

Service Employment:

The percentage of residents working in service employment ranged from 1.9-16.9\%. We calibrated service employment based on tertiles where values $\leq 7.9 \%$ were coded as zero (low service employment), values from $7.9-9.8 \%$ were coded as one (medium service employment), and values greater than $9.8 \%$ were coded as two (high service employment).

Overcrowding:

Percentages of overcrowding ranged from 0-6.1\%. We calibrated overcrowding based on tertiles where values $\leq 1.7 \%$ were coded as zero (low overcrowding), values from $1.7-2.4 \%$ were coded as one (medium overcrowding), and values $>2.4 \%$ were coded as 2 (high overcrowding).

Meta-factor Calibrations:

We also created meta-factors using dual calibrations of each factor to identify patterns among potential difference makers among the conditions. For example, for education, we calibrated a factor called high 
education where neighborhoods in the highest tertile of education were coded as one, and those not in the highest tertile (low to medium) were coded as zero. We also created a factor called low education where neighborhoods in the lowest tertile of education were coded as one and those not in the lowest tertile (medium to high) were coded as zero.

\section{Factor Selection:}

To reduce our data and focus our analysis, we implemented a configurational approach to factor selection described in detail elsewhere (26-30). Briefly, we began by using the "minimally sufficient conditions" (i.e., msc function within the R package "cna") to look across all 43 neighborhoods and all 8 factors at once, comprehensively scanning the entire dataset to identify specific configurations of conditions with strong connections to the outcome of interest (i.e., persistently low COVID-19 vaccination rates). This process exhaustively considered all one-, two- and three-condition configurations instantiated in the dataset, assessed each configuration against a prespecified consistency threshold, retained all configurations that satisfied this criterion, and then generated a "condition table" to list and organize the Boolean output. In a condition table, rows contain all configurations of conditions that meet a specified consistency level while column variables include outcome, conditions, consistency and coverage. We generated the msc routine condition tables by specifying a consistency threshold of $100 \%$; if no configurations met this threshold, we iteratively lowered the specified consistency level by 5 points (e.g., from $100-95 \%$, etc.) and repeated the process to generate a new condition table. We continued lowering the consistency threshold until there were at least two potential configurations of neighborhood-level conditions that met the specified consistency level. Using this approach, we inductively analyzed the entire dataset and used the condition table output to identify a subset of candidate factors to use in model development in the next steps of the configurational analysis.

Model Development:

We next developed models by iteratively using model-building functions within the R "cna" software package. We assessed candidate models based on their overall consistency and coverage, as well as potential model ambiguity (when competing models satisfy the specified consistency and coverage thresholds and explain the outcome of interest equally well, as reflected by similar consistency and coverage scores). We selected a final model based on the same criteria of overall consistency and coverage, with no model ambiguity. The Coincidence Analysis package ("cna") in R (32), R (version 3.5.0), and Microsoft Excel were used to support the analyses. Maps were created with ArcGISPro 2.9.1 (ESRI, Redlands CA) using 2010 ZIP Code Tabulation Area (ZCTA) boundaries from the US Census.

\section{Results}

Two factors consistently distinguished neighborhoods with persistently low COVID-19 vaccination rates from the others: education and concentrated racial privilege. The combination of low college education together with low/medium concentrated racial privilege was sufficient to identify neighborhoods with persistently low COVID-19 vaccination rates. 
In the geospatial mapping of these findings (see Fig. 1), one can directly observe that all but two of the neighborhoods with persistently low COVID-19 vaccination rates (i.e., the neighborhoods with zip codes listed) have a combination of green and brown, indicating the joint presence of low college education and low/medium concentrated racial privilege. This solution thus explains or "covers" 11 of the 13 neighborhoods with persistently low COVID-19 vaccination rates, translating into a coverage score of .85 $(11 / 13)$. Furthermore, the solution is highly consistent in distinguishing neighborhoods with persistently low COVID-19 vaccination rates from the others, as there is only neighborhood in the analysis (zip code 19121, directly below 19132, labeled on the map) that had both conditions present and did not have persistently low COVID-19 vaccination rates, translating into a consistency score of .92 (11/12). Featuring both high coverage and consistency scores, the combination of these two specific conditions proves a crucial difference-maker.

We also explored how levels of college education and/or concentrated racial privilege linked to those 29 neighborhoods where COVID-19 vaccination rates were not persistently low. Medium/high college education by itself was a sufficient condition for not being a neighborhood with persistently low neighborhood COVID-19 vaccination rates, as was high concentrated racial privilege.

The geospatial mapping of these findings (see Fig. 2) shows that every neighborhood with medium/high COVID-19 vaccination rates (i.e., the neighborhoods with zip codes listed) has either green dots, brown dots, or a combination of green and brown dots, with only one exception. This solution thus explains or "covers" 28 of the 29 neighborhoods without persistently low vaccination rates, yielding an extremely high coverage score of .97 (28/29). The solution is also highly consistent in distinguishing neighborhoods with medium/high vaccination rates from the others, as there are only two neighborhoods in the analysis that had either condition present and did not have medium/high vaccination rates for a coverage score of .93 (28/30). With both high coverage and consistency scores, the presence of either medium/high college or high concentrated racial privilege are difference-making conditions that uniquely and consistently identified neighborhoods with medium/high COVID-19 vaccination rates.

\section{Discussion}

Our study used CCMs, specifically CNA (22), along with geospatial mapping, to identify how multiple neighborhood conditions link to persistently low COVID-19 vaccination rates. A key finding from this study is that while neither low college education nor low/medium concentrated racial privilege alone was linked with persistently low COVID-19 vaccination rates, the presence of both these factors together was consistently linked to this outcome. Our complementary analysis of neighborhoods that do not have persistently low COVID-19 vaccination rates affirms the importance of these same two factors: education and racial privilege. We found that different values of these two factors, in other words medium/high college education and high concentrated racial privilege, were consistently linked to neighborhoods without persistently low COVID-19 vaccination rates, with the presence of either condition alone proving sufficient. 
Our study adds a new perspective to how social conditions impact COVID-19 vaccination rates. We found that in eleven of thirteen neighborhoods, the combination of low education and low/medium concentrated racial privilege was consistently linked to persistently low COVID-19 vaccination rates. The finding that the combination of multiple factors was linked to low COVID-19 vaccination rates demonstrates the concept of Boolean conjunction, where multiple conditions together are sufficient to yield an outcome. We found a different pattern for the neighborhoods without persistently low COVID-19 vaccination rates, where either factor alone - medium/high college education or high concentrated racial privilege - was linked to higher COVID-19 vaccination rates. This finding that multiple different factors taken alone were each sufficient to produce the outcome demonstrates the concept of Boolean disjunction.

In this analysis, low/medium concentrated racial privilege by itself did not link directly to persistently low COVID-19 vaccination rates, but it did when combined with low levels of college education. This is consistent with previous studies showing that COVID-19 vaccination rates vary by socioeconomic factors within racial/ethnic groups $(33,34)$. Residential segregation is known to have a strong link to poverty, poor health, and lack of access to health care (35-37). Williams and Collins have found that segregation exerts its negative effect on individual and neighborhood health not only through socioeconomic status, but also through the effects of place, shaping health behaviors, poorer access to medical care, neighborhood quality and crime, and lower quality education (37). At the neighborhood level, poorer access to medical care may have included lack of access to clinics or pharmacies where COVID-19 vaccines were available. In Philadelphia, it was this very lack of health care access that spurred the launch of the Black Doctors COVID Consortium to address issues of access and mistrust by providing COVID testing and vaccinations at community sites, including churches (38). Studies using a combined measure of residential segregation and income inequity, found that all three factors - racial segregation, economic segregation and racialized economic segregation - were associated with poor COVID-19 outcomes (39). We included a measure of economic privilege - the Index of Concentration at the Extremes - Income - but this factor was not sufficient alone or in combination with other factors to link to low COVID-19 vaccination rates.

Beyond its direct impact on access to health care, residential racial segregation degrades the quality of schools, causing both individual and community-wide impacts (37). Low levels of education are also known to have strong association with poor health outcomes. Kawachi and others have suggested that at the individual level, education provides general knowledge that may be helpful in preventing disease, but that it also confers greater status and greater access to jobs that pay well and have fewer safety hazards (40). The findings in our study align with other research demonstrating that low levels of education negatively impact vaccine acceptance and health status overall at the individual level (15, 41, 42) and at the community level (43).

A strength of our study is the ability to uncover causal complexity using a CNA, a novel configurational analytic method. This method reveals findings that are complementary to results from studies using correlational methods and helps to uncover qualitative differences between neighborhoods that may be 
important to designing interventions. To our knowledge, this is the first study to apply CCMs, specifically CNA, to a geospatial analysis of the important public health and health disparity challenge of low COVID19 vaccination rates. It is our hope that future studies seeking to understand health disparities will employ similar methods.

Our findings align with the concept of intersectionality, a framework for understanding "how multiple social identities such as race, gender, sexual orientation, SES, and disability intersect at the micro level of individual experience to reflect interlocking systems of privilege and oppression (i.e., racism, sexism, heterosexism, classism) at the macro social structural level (44)." Original concepts of intersectionality emerged from feminist theory and increasingly, its theoretical and methodological concepts are being applied to the ways that public health scholars study complex health inequities. As the conceptual and methodological applications of intersectionality continue to grow, a number of scholars have called for intersectional approaches to address pressing health equity challenges such as COVID-19 $(45,46)$. In our study, by emphasizing the ways that disadvantages and advantages intersect, we are able to paint a more complex picture in ways that improve our ability to diagnose and address social inequities $(18,47)$.

Our study has several weaknesses. One weakness of this study is the use of vaccination data at the zip code tabulation area level. ZCTAs (and ZIP Codes) represent large and relatively heterogeneous geospatial units of analysis which do not necessarily correspond to the neighborhoods that residents might define, nor do they drill down to the level of census tracts. Future research performed at the census tract level or at the community resident-defined neighborhood level is needed to better understand complex drivers of health inequities at the neighborhood level. A second potential limitation is that while our data refers to neighborhood (ZCTA) of residence, people vaccinated in other states may not be included in this dataset due to the lack of federal coordination in vaccination data systems.

In Philadelphia from December 16, 2020, through April 12, 2021, eligibility for COVID-19 vaccinations was limited to higher risk groups, such as health care workers, essential workers, those over the age of 65 and those with chronic conditions. On April 16, 2021, eligibility was expanded to include all residents 16 years of age or older. This raises the potential limitation that neighborhoods with persistently low COVID-19 vaccination rates might have had a lower proportion of eligible individuals than other neighborhoods. While we did not include factors reflecting proportions of populations in various high-risk groups, we found that that the neighborhood factors that were linked with persistently low COVID-19 vaccination rates in March and April were also linked to persistently low COVID-19 vaccination rates in May after eligibility was expanded to all residents.

We included factors that related to COVID-19 infection rates and mortality at the neighborhood level in previous studies. It is possible that other factors relating to COVID-19 vaccination rates in specific (e.g., trust in governmental institutions or ideology) (41) and which might be revealed through in-depth qualitative studies, were not included in this analysis. Nonetheless, the combinations of conditions and the causal pathways identified here were able to identify neighborhoods with low COVID-19 vaccination 
rates with high consistency and coverage. Future studies using qualitative and quantitative methods will be useful in defining the underlying mechanisms necessary for intervention.

\section{Abbreviations}

CCM - configurational comparative methods

CNA- coincidence analysis

ICE-Income - Index of Concentration at the Extremes - Income

ICE-BlackNH - Index of Concentration at the Extremes - Non-Hispanic Black

COVID-19 - Coronavirus disease 2019

\section{Conclusions}

A practical policy implication of these findings is that neighborhoods that have low rates of COVID-19 vaccination and other pressing health disparities, may require strategies designed to address multiple intersecting factors. For example, in neighborhoods where low levels of education combine with low concentrated racial privilege, health enhancing interventions should focus on improving access to higher education while also dismantling the structural racism underlying residential segregation. Approaches that use CCMs to identify geospatial patterns, which might best be termed "geoconfigurational," represent a unique approach to understanding health inequities at the neighborhood level. This approach affirms the credo that "social policy is health policy." The complex interplay of specific social vulnerabilities must be better understood and addressed with the same vigor as individually focused interventions aimed at increasing COVID-19 vaccination rates. Future research should also shed light on how these pathways relate to disparities in other health outcomes, such as violence and chronic disease. The results of such studies could guide public health policymakers and community leaders in addressing the challenges that are most specific to their communities.

\section{Declarations}

\section{Ethics approval and consent to participate:}

The Institutional Review Board at Drexel University reviewed the protocol, determined that the use of publicly available de-identified data is not human subjects research, and has waived informed consent. All data analyzed for this study were anonymous and publicly available in aggregate form. All methods were carried out in accordance with relevant guidelines and regulations.

Consent for publication - Not applicable

Availability of data and materials: 
Datasets and code for replication are available at

https://github.com/JohnARich/CNA_COVIDVax_Philly

Competing interests:

The authors declare that they have no competing interests.

\section{Funding:}

UB is partially supported by the Office of the Director of the National Institutes of Health under award number DP5OD26429. The other authors have no funding sources to declare.

\section{Contributions:}

JAR: conception, data collection, design of the work, data analysis and interpretation, drafting the article, critical revisions to the article. EJM: data analysis and interpretation, drafting the article, critical revisions to the article. UB: data collection, data analysis and interpretation, critical revisions to the article. TJC: design of the work, data analysis and interpretation, drafting the article, critical revisions to the article. All authors read and approved the final manuscript.

Acknowledgements:

The authors would like to acknowledge Steven Melly, GIS Analyst, Urban Health Collaborative, Dornsife School of Public Health at Drexel University for his assistance in creating the maps for the manuscript, and Danny Galpern, Philadelphia Department of Public Health for his assistance in data acquisition and management.

\section{Author information:}

\section{Affiliations:}

Department of Health Management and Policy, Dornsife School of Public Health at Drexel University, Philadelphia, PA.

John A. Rich

William M. Tierney Center for Health Services Research, Regenstrief Institute, Indianapolis, IN.

\section{Edward J. Meich}

Department of Epidemiology and Biostatistics, and Urban Health Collaborative at the Dornsife School of Public Health at Drexel University, Philadelphia, PA

Usama Bilal 
Department of Emergency Medicine, Rush University Medical Center, Chicago, IL

Theodore J. Corbin

\section{References}

1. Shah M, Sachdeva M, Dodiuk-Gad RP. COVID-19 and racial disparities. Journal of the American Academy of Dermatology. 2020;83(1):e35.

2. Yancy CW. COVID-19 and african americans. Jama. 2020;323(19):1891-2.

3. Bilal U, Tabb LP, Barber S, Diez Roux AV. Spatial Inequities in COVID-19 Testing, Positivity, Confirmed Cases, and Mortality in 3 US Cities: An Ecological Study. Annals of internal medicine. 2021.

4. DiMaggio $C$, Klein M, Berry C, Frangos S. Black/African American Communities are at highest risk of COVID-19: spatial modeling of New York City ZIP Code-level testing results. Annals of epidemiology. 2020;51:7-13.

5. Injustice in Health: Now Is the Time to Change the Story. Annals of Internal Medicine. 2021;174(7):1012-3.

6. National Academies of Sciences E, Medicine. Framework for Equitable Allocation of COVID-19 Vaccine. Gayle H, Foege W, Brown L, Kahn B, editors. Washington, DC: The National Academies Press; 2020. 272 p.

7. Barry V, Dasgupta S, Weller DL, Kriss JL, Cadwell BL, Rose C, et al. Patterns in COVID-19 Vaccination Coverage, by Social Vulnerability and Urbanicity-United States, December 14, 2020-May 1, 2021. Morbidity and Mortality Weekly Report. 2021;70(22):818.

8. Hughes MM, Wang A, Grossman MK, Pun E, Whiteman A, Deng L, et al. County-level COVID-19 vaccination coverage and social vulnerability-United States, December 14, 2020-March 1, 2021. Morbidity and Mortality Weekly Report. 2021;70(12):431.

9. Whiteman A, Wang A, McCain K, Gunnels B, Toblin R, Lee JT, et al. Demographic and social factors associated with COVID-19 vaccination initiation among adults aged $\geq 65$ years-United States, December 14, 2020-April 10, 2021. Morbidity and Mortality Weekly Report. 2021;70(19):725.

10. Philadelphia Department of Public Health Vaccination Dashboard [cited 2021 June 30]. Available from: https://www.phila.gov/programs/coronavirus-disease-2019-covid-19/data/vaccine/.

11. Bilal U, Mullachery P, Schnake-Mahl A, McCulley E, Kolker J, Barber S, et al. Heterogeneity in the Spatial Inequities in COVID-19 Vaccination in across 16 Large US Cities. American journal of epidemiology. 2022; In Press.

12. Anaele BI, Doran C, Mclntire R. Visualizing COVID-19 mortality rates and African-American populations in the USA and Pennsylvania. Journal of Racial and Ethnic Health Disparities. 2021:1-8.

13. Barber S, Headen I, Branch B, Tabb L, Yadeta K. COVID-19 in Context: Racism, Segregation and Racial Inequities in Philadelphia. Drexel University Urban Health Collaborative. 2020. 
14. Chen JT, Krieger N. Revealing the unequal burden of COVID-19 by income, race/ethnicity, and household crowding: US county versus zip code analyses. Journal of Public Health Management and Practice. 2021;27(1):S43-S56.

15. Barry V, Dasgupta S, Weller DL, Kriss JL, Cadwell BL, Rose C, et al. Patterns in COVID-19 Vaccination Coverage, by Social Vulnerability and Urbanicity - United States, December 14, 2020-May 1, 2021. MMWR Morbidity and Mortality Weekly Report. 2021;70(22):818-24.

16. Hughes MM, Wang A, Grossman MK, Pun E, Whiteman A, Deng L, et al. County-Level COVID-19 Vaccination Coverage and Social Vulnerability - United States, December 14, 2020-March 1, 2021. MMWR Morbidity and Mortality Weekly Report. 2021;70(12):431-6.

17. Gaynor TS, Wilson ME. Social Vulnerability and Equity: The Disproportionate Impact of COVID-19. Public Administration Review. 2020;80(5):832-8.

18. Ragin CC, Fiss PC. Intersectional inequality: Race, class, test scores, and poverty: University of Chicago Press; 2017.

19. Ragin CC. Comparative Method: Moving Beyond Qualitative and Quantitative Strategies 2014. 1-185 p.

20. Whitaker RG, Sperber N, Baumgartner M, Thiem A, Cragun D, Damschroder L, et al. Coincidence analysis: a new method for causal inference in implementation science. Implementation Science. 2020;15(1):1-10.

21. Hanckel B, Petticrew M, Thomas J, Green J. The use of Qualitative Comparative Analysis (QCA) to address causality in complex systems: a systematic review of research on public health interventions. BMC Public Health. 2021;21(1):877.

22. Baumgartner $M$, Ambühl M. Causal modeling with multi-value and fuzzy-set Coincidence Analysis. Political Science Research and Methods. 2020;8(3):526-42.

23. Schneider CQ, Wagemann C. Set-theoretic methods for the social sciences: A guide to qualitative comparative analysis: Cambridge University Press; 2012.

24. Shi C, Liao L, Li H, Su Z. Which urban communities are susceptible to COVID-19? An empirical study through the lens of community resilience. BMC Public Health. 2022;22(1):70.

25. Ragin CC. The comparative method: University of California Press; 2014.

26. Cohen DJ, Sweeney SM, Miller WL, Hall JD, Miech EJ, Springer RJ, et al. Improving smoking and blood pressure outcomes: the interplay between operational changes and local context. The Annals of Family Medicine. 2021;19(3):240-8.

27. Coury J, Miech EJ, Styer P, Petrik AF, Coates KE, Green BB, et al. What's the "secret sauce"? How implementation variation affects the success of colorectal cancer screening outreach. Implementation Science Communications. 2021;2(1):1-11.

28. Hickman SE, Miech EJ, Stump TE, Fowler NR, Unroe KT. Identifying the implementation conditions associated with positive outcomes in a successful nursing facility demonstration project. The Gerontologist. 2020;60(8):1566-74. 
29. Petrik AF, Green B, Schneider J, Miech EJ, Coury J, Retecki S, et al. Factors influencing implementation of a colorectal cancer screening improvement program in community health centers: an applied use of Configurational Comparative Methods. Journal of General Internal Medicine. 2020;35(2):815-22.

30. Yakovchenko V, Miech EJ, Chinman MJ, Chartier M, Gonzalez R, Kirchner JE, et al. Strategy configurations directly linked to higher hepatitis $C$ virus treatment starts: an applied use of configurational comparative methods. Medical care. 2020;58(5):e31.

31. Krieger N, Waterman PD, Spasojevic J, Li W, Maduro G, Van Wye G. Public health monitoring of privilege and deprivation with the index of concentration at the extremes. American journal of public health. 2016;106(2):256-63.

32. Ambühl M, Baumgartner M, Epple R, Thiem A. Cna: causal modeling with coincidence analysis. 2020.

33. Green-McKenzie J, Shofer FS, Momplaisir F, Kuter BJ, Kruse G, Bialal U, et al. Factors Associated With COVID-19 Vaccine Receipt by Health Care Personnel at a Major Academic Hospital During the First Months of Vaccine Availability. JAMA network open. 2021;4(12):e2136582-e.

34. Thomas K, Darling J, Cassil A. Education Is Now a Bigger Factor Than Race in Desire for COVID-19 Vaccine.. Los Angeles, California: USC Dornsife Center for Economic and Social Research; 2021.

35. Snowden LR, Graaf G. COVID-19, social determinants past, present, and future, and African Americans' health. Journal of racial and ethnic health disparities. 2021;8(1):12-20.

36. White K, Borrell LN. Racial/ethnic residential segregation: Framing the context of health risk and health disparities. Health \& Place. 2011;17(2):438-48.

37. Williams DR, Collins C. Racial Residential Segregation: A Fundamental Cause of Racial Disparities in Health. Public Health Reports. 2001;116(5):404-16.

38. Jaklevic MC. Surgeon Fills COVID-19 Testing Gap in Philadelphia's Black Neighborhoods. JAMA. 2021;325(1):14-6.

39. Brown KM, Lewis JY, Davis SK. An ecological study of the association between neighborhood racial and economic residential segregation with COVID-19 vulnerability in the United States' capital city. Annals of Epidemiology. 2021;59:33-6.

40. Kawachi I, Adler NE, Dow WH. Money, schooling, and health: Mechanisms and causal evidence. Annals of the New York Academy of Sciences. 2010.

41. Agarwal R, Dugas M, Ramaprasad J, Luo J, Li G, Gao GG. Socioeconomic privilege and political ideology are associated with racial disparity in COVID-19 vaccination. Proceedings of the National Academy of Sciences. 2021;118(33).

42. Fisher KA, Bloomstone SJ, Walder J, Crawford S, Fouayzi H, Mazor KM. Attitudes toward a potential SARS-CoV-2 vaccine: a survey of US adults. Annals of internal medicine. 2020;173(12):964-73.

43. Sacarny A, Daw JR, editors. Inequities in COVID-19 vaccination rates in the 9 largest US cities. JAMA Health Forum; 2021: American Medical Association. 
44. Bowleg L. The problem with the phrase women and minorities: intersectionality-an important theoretical framework for public health. American journal of public health. 2012;102(7):1267-73.

45. Bowleg L. Evolving Intersectionality Within Public Health: From Analysis to Action. American Journal of Public Health. 2021;111(1):88-90.

46. Elnaiem AD. Intersectionality in the Time of COVID-19: Dispatches From a Contact Tracer. American Journal of Public Health. 2021;111(1):93-4.

47. Hancock A-M. When multiplication doesn't equal quick addition: Examining intersectionality as a research paradigm. Perspectives on politics. 2007;5(1):63-79.

\section{Figures}

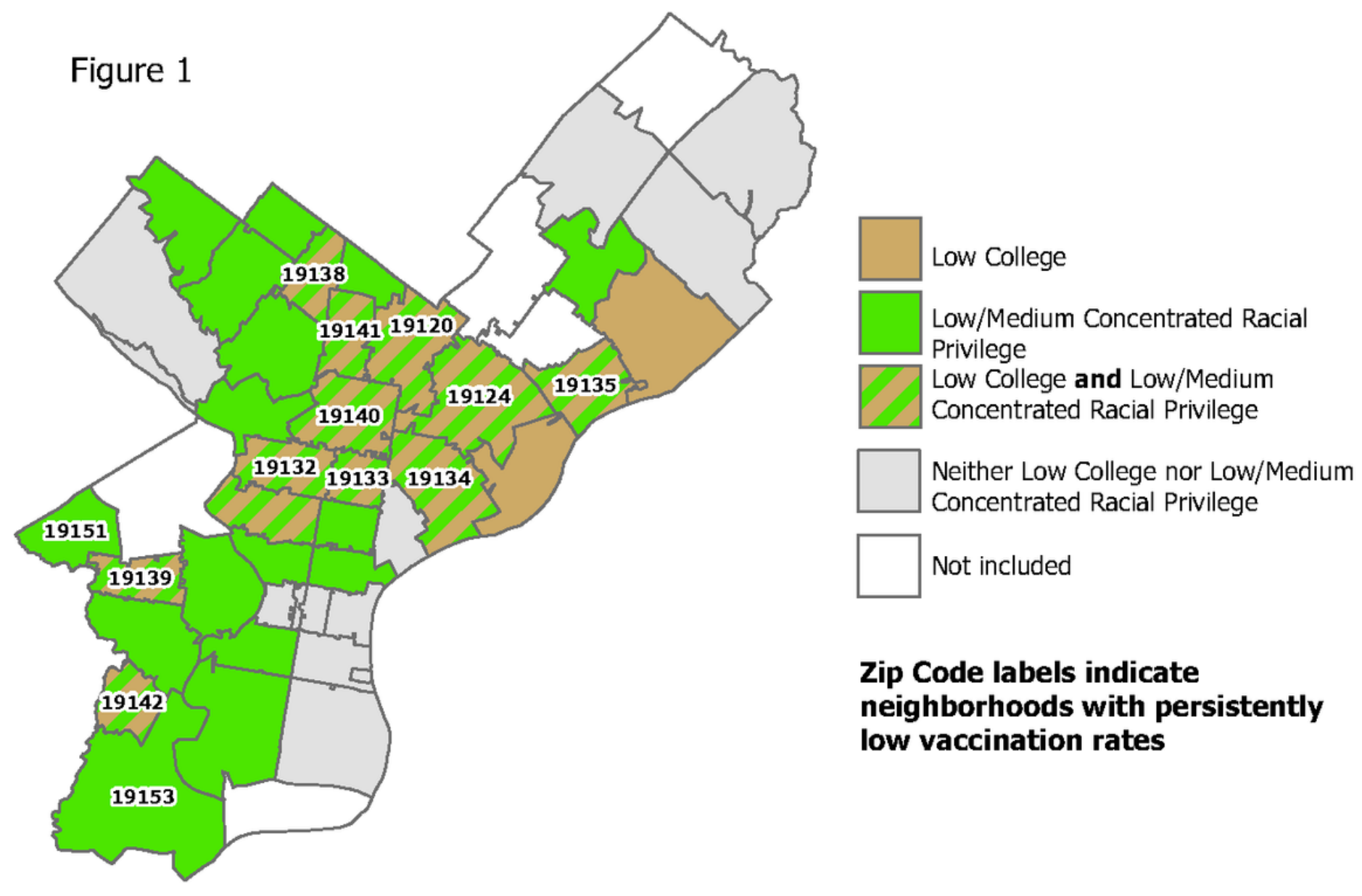

Figure 1

Pathways sufficient for neighborhoods having persistently low COVID-19 vaccination rates by neighborhood 


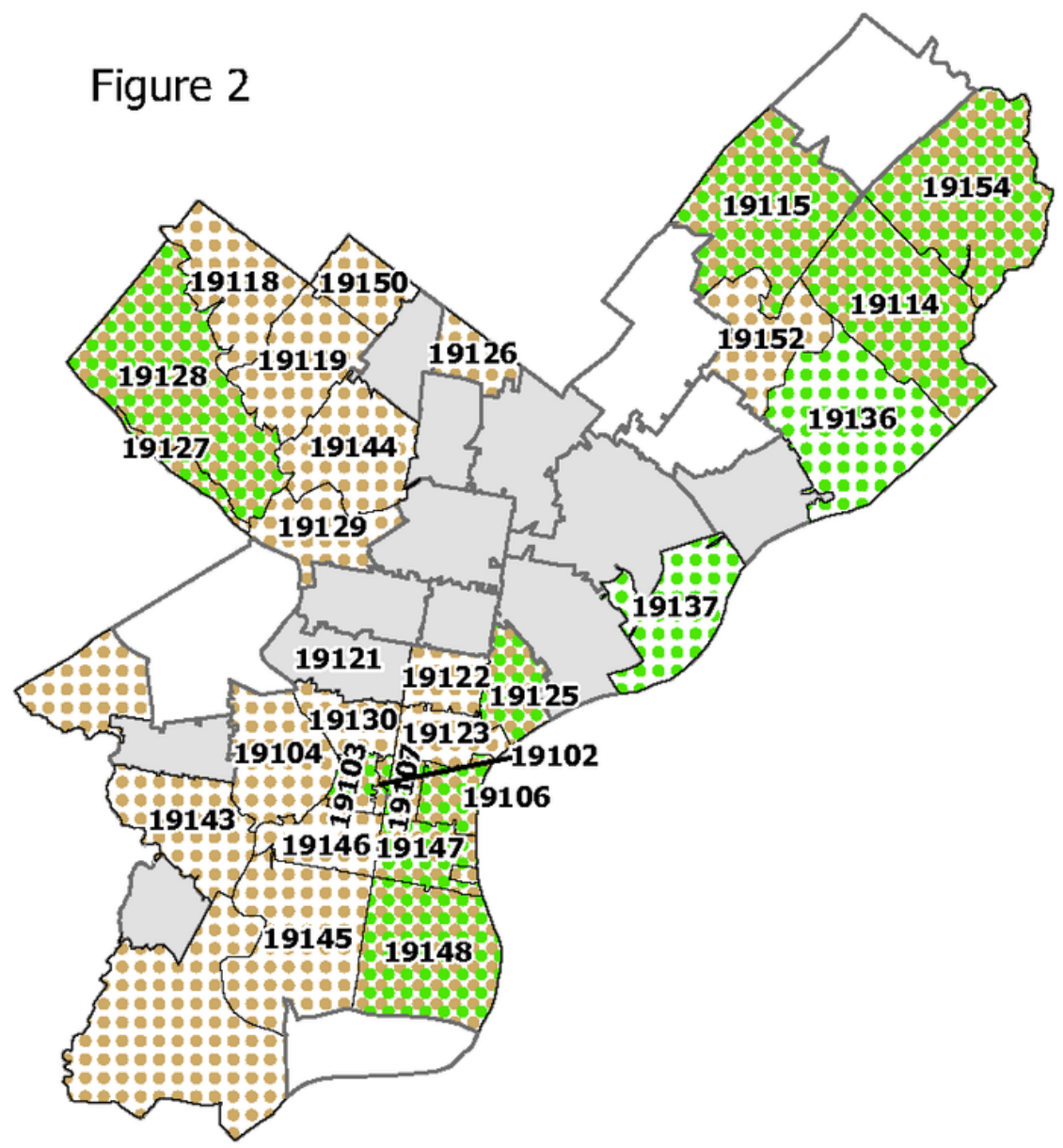

10. Medium/High College
10.8 High Concentrated Racial Privilege
58 Medium/High College and
High Concentrated Racial Privilege

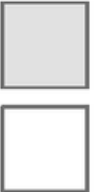

Neither Medium/High College nor High Concentrated Racial Privilege

Not included

\section{Zip code labels indicate neighborhoods without persistently low vaccination rates}

Figure 2

Pathways sufficient for neighborhoods not having persistently low COVID-19 vaccination rates by neighborhood 